\title{
Auto-Regulação: Diferenças em Função do Ano e Área em Alunos Universitários
}

\author{
Iolanda da Silva Ribeiro ${ }^{1}$ \\ Carla Ferreira da Silva \\ Universidade do Minho
}

\begin{abstract}
RESUMO - Neste estudo procura-se analisar a existência de diferenças nas estratégias auto-regulatórias de alunos universitários em áreas de formação distintas. Participaram 518 alunos de três níveis (inicial, intermédio e final) das áreas de ciências e humanidades. Aplicou-se a escala "CHE - Comportamentos e hábitos de estudo e aprendizagem", que avalia cinco dimensões: estratégias cognitivas de transformação e manipulação da informação, organização e planeamento de rotinas, gestão e monitorização, aquisição e selecção da informação, e reforço motivacional. Verificou-se uma maior utilização das estratégias cognitivas e metacognitivas de gestão e monitorização apesar dos resultados não indicarem diferenças substantivas entre os alunos diferenciados por nível e área. Os resultados podem indicar estabilidade nos comportamentos ou limitações no tipo de instrumento e amostra utilizada. $\mathrm{O}$ estudo de mudanças nestas estratégias deverá ser conduzido com recurso a delineamentos longitudinais. O impacto da estabilidade deverá ser ponderado na elaboração de projectos de intervenção.
\end{abstract}

Palavras-chave: auto-regulação; aprendizagem; ensino superior.

\section{Self-Regulation: Differences by Year and Area in College Students}

\begin{abstract}
In this study we seek to analyze the existence of differences in self-regulating strategies of university students in distinct graduation areas. 518 students of three levels (initial, intermediate and final) of science and humanities fields participated. We used the scale "Behavior and study skill", which evaluates five dimensions: cognitive strategies of transformation and manipulation of information, organization and planning of routines, management and monitoring, information acquisition and selection, and motivational reinforcement. A higher use of cognitive and metacognitive strategies of management and monitoring was noted although the results do not indicate major differences between students in different levels and graduation areas. The results can indicate stability in the behaviors or limitations in the type of instrument and in the sample used. The study of changes in these strategies must be carried out having in mind longitudinal outlines. The impact of the stability should be taken into account while elaborating intervention projects.
\end{abstract}

Key words: self-regulation; learning; graduate teaching.

No ensino superior, é exigido aos alunos uma autonomia considerável no seu estudo (Almeida, 2001), o que implica a utilização deliberada de estratégias activas de aprendizagem. A utilização eficaz de competências que permitam interpretar e compreender a informação, a resolução de problemas, a reflexão crítica e a generalização de conceitos, a formulação de questões, e a expressão oral e escrita são cruciais (Read, 1999), uma vez que constituem planos de acção que ajudam os alunos a adquirir e codificar a informação, bem como a executar, de modo optimizado e autónomo, as várias tarefas escolares (Zimmerman, 2001; Zimmerman \& MartinezPons, 1992).

No entanto, com frequência, verifica-se que os alunos não só não revelam hábitos de estudo, como não são capazes de fazer uma abordagem adequada no sentido de seleccionar as estratégias de aprendizagem em função da especificidade dos conteúdos e exigências das diferentes matérias curriculares. Assim, a capacidade de auto-regulação do aluno revela-se como um factor importante no seu sucesso académico (Bes-

1 Endereço: Instituto de Educação e Psicologia, Campus de Gualtar, Braga, Portugal 4710-057. E-mail: iolanda@iep.uminho.pt sa, 2000; Santos, 2001), caracterizando-se por uma postura activa dos alunos no processo de aprendizagem, a um nível metacognitivo, motivacional e comportamental (Zimmerman, 2001). O aluno auto-regulado está consciente e controla o seu processo de aprendizagem; selecciona os métodos e as estratégias que utiliza, revelando um grande sentido de autoeficácia; e organiza e estrutura quer o seu contexto de estudo quer o trabalho a realizar, identificando as situações em que precisa de ajuda e adaptando as estratégias de aprendizagem aos seus objectivos académicos (Wolters, 1998; Zimmerman, 1994). O aluno auto-regulado caracteriza-se por estabelecer objectivos de aprendizagem, ter consciência das suas capacidades e limitações, e perspectivar todo o processo de aprendizagem como algo que pode controlar desde a procura da informação até as estratégias a utilizar, responsabilizandose pelos resultados obtidos (Borkowski, Carr, Rellinger \& Pressley, 1990; Pintrich, 2000; Zimmerman, 1994, 1998).

A relação entre estratégias de aprendizagem, auto-regulação e rendimento escolar foi evidenciada em diversos estudos em Portugal. Bessa (2000) analisou os níveis de ajustamento e de auto-regulação académica em alunos do primeiro ano do ensino superior e verificou o recurso sistemático a estratégias auto-reguladoras da aprendizagem, e a existência de 
uma relação entre esta utilização e os níveis de ajustamento académico (nomeadamente, o grau de confiança em si e a satisfação com a universidade). Recorrendo igualmente a uma amostra de alunos do $1^{\circ}$ ano, Santos (2001) verificou que o sucesso e a adaptação académica se encontravam relacionados, entre outros, com a utilização de métodos de estudo. Recentemente, em vários estudos tem sido analisada a relação da auto-regulação com a procrastinação, verificando-se que sujeitos que evidenciavam comportamentos de procrastinação revelavam uma menor capacidade de regulação do seu desempenho (Dewitte, 2000; Ferrari, 2001, Howell, Watson, Powell \& Buro, 2006). Por sua vez, Howell e Watson (2007) verificaram que a utilização de estratégias cognitivas e metacognitivas é muito reduzida nos sujeitos com este tipo de comportamentos.

Os estudos desenvolvidos por Brown e colaboradores na década de 1980 (Brown \& Day, 1983; Brown, Day \& Jones, 1983) indicam que a auto-regulação se desenvolve de modo lento e que os alunos universitários demonstram ser capazes de usarem competências auto-regulatórias no estudo. Porém, estes resultados têm sido questionados por alguns autores (Peverly, Brobst, Graham \& Shaw, 2003; Pressley \& Ghatala, 1990; Pressley, Ghatala, Woloshyn \& Pirie, 1990), os quais sugerem que os alunos universitários nem sempre são capazes de usar estratégias auto-regulatórias, registando várias dificuldades na sua utilização. No entanto, apesar de actualmente vários autores se debruçarem, nos seus estudos, sobre a importância da aprendizagem auto-regulada no ensino superior, têm sido encontradas algumas limitações relativamente à população estudada, uma vez que esta se tem centrado em alunos de uma fase inicial deste nível de ensino. Neste estudo, procuramos verificar se existem alterações nos padrões de utilização das estratégias auto-reguladoras da aprendizagem dos alunos, quer durante a sua frequência no ensino superior, quer em áreas de formação distintas.

\section{Método}

\section{Participantes}

O estudo inclui uma amostra de 518 alunos da Universidade do Minho, distribuídos por três níveis: inicial (alunos do $1^{\circ}$ ano), intermédio (alunos do $2^{\circ}$ e $3^{\circ}$ ano), e final (alunos do $4^{\circ}$ ano), inscritos em cursos das áreas de ciências (Física e Química e Engenharia Civil) e humanidades (Português e Relações Internacionais). A percentagem de alunos do género feminino é mais elevada nas duas áreas (cf. Tabela 1). Esta

Tabela 1. Descrição da amostra.

\begin{tabular}{|c|c|c|c|c|c|c|c|}
\hline \multirow[b]{2}{*}{ Grupo } & \multirow[b]{2}{*}{$n$} & & \multicolumn{2}{|c|}{ Género } & \multicolumn{2}{|c|}{ Idade } & \multirow[b]{2}{*}{ Amplitude } \\
\hline & & & $n$ & $\%$ & $M$ & $D P$ & \\
\hline \multirow{2}{*}{$\begin{array}{l}\text { Amostra } \\
\text { Global }\end{array}$} & \multirow{2}{*}{518} & Masc. & 179 & 34,6 & 21,8 & 3,49 & $18-42$ \\
\hline & & Fem. & 339 & 65,4 & 21,2 & 2,76 & $18-38$ \\
\hline \multirow{2}{*}{ Ciências } & \multirow{2}{*}{274} & Masc. & 121 & 44,2 & 22,1 & 3,82 & $18-42$ \\
\hline & & Fem. & 153 & 55,8 & 21,5 & 2,81 & $18-38$ \\
\hline \multirow{2}{*}{ Letras } & \multirow{2}{*}{244} & Masc. & 58 & 23,8 & 21,3 & 2,64 & $18-31$ \\
\hline & & Fem. & 186 & 76,2 & 20,8 & 2,68 & $18-38$ \\
\hline
\end{tabular}

distribuição traduz a real distribuição de alunos encontrada, actualmente, no ensino superior português.

A média de idades nos alunos universitários do sexo feminino e masculino é similar, as diferenças de idade observadas para os alunos em função dos cursos não é estatisticamente significativa. O número de alunos por nível é diferente, como se pode observar na Tabela 2. A maior frequência no nível intermédio reflecte a inclusão neste grupo de alunos do segundo e terceiro ano. A diferença no número de alunos nos níveis iniciais e finais reflecte a redução substancial no número de candidatos, observada nos últimos anos, ao ensino superior português nos cursos que habilitam para a docência. Esta redução encontra-se relacionada com a redução substancial na taxa de natalidade existente no país, que já se reflectiu, em anos anteriores, nos níveis de ensino básico e secundário e que começa agora a afectar o ensino superior. Os cursos de Física e Química e de Português considerados neste estudo reflectem esta tendência.

Tabela 2. Descrição dos participantes por nível e área.

\begin{tabular}{lccccccc}
\hline & & \multicolumn{2}{c}{ Inicial } & \multicolumn{2}{c}{ Intermédio } & \multicolumn{2}{c}{ Final } \\
\cline { 3 - 8 } Grupo & $n$ & $n$ & $\%$ & $n$ & $\%$ & $n$ & $\%$ \\
\hline $\begin{array}{l}\text { Amostra } \\
\text { Global }\end{array}$ & 518 & 131 & 25,3 & 216 & 41,7 & 171 & 33 \\
Ciências & 274 & 46 & 16,8 & 125 & 45,6 & 103 & 37,6 \\
Letras & 244 & 85 & 34,8 & 91 & 37,3 & 68 & 27,9 \\
\hline
\end{tabular}

\section{Instrumentos}

Para a avaliação das estratégias de aprendizagem autoreguladas recorreu-se à escala "CHE - Comportamentos habituais de estudo e aprendizagem" (Bessa \& Tavares, 2001). Esta escala foi desenvolvida na Universidade de Aveiro e serviu de base a um estudo que procurou verificar a relação entre níveis de ajustamento académico, abordagens e estratégias de auto-reguladoras da aprendizagem. A escala é constituída por 25 itens com resposta de formato tipo Likert de cinco pontos (01 para o valor mínimo/discordância total e o 05 para o valor máximo/concordância total). Os itens distribuem-se por cinco factores que correspondem a cinco tipos de estratégias de auto-regulação. $\mathrm{O}$ factor 1 corresponde às "estratégias cognitivas de transformação e manipulação da informação" (ECTMI, sete itens) estando relacionado com a distinção e selecção da informação e com a sua conexão para posterior interiorização. $O$ factor 2 corresponde às "estratégias cognitivas de organização e planeamento de rotinas" (ECOPR, cinco itens) e está relacionado com a assiduidade e pontualidade em relação à frequência às aulas, com o tempo dedicado ao estudo e com a utilização de dossiers de apontamentos e dos materiais necessários ao estudo. $\mathrm{O}$ factor 3 corresponde às "estratégias cognitivas e metacognitivas de gestão e monitorização" (ECMGM, sete itens) onde se englobam a gestão e o controlo da informação, a consciencialização dos distractores de estudo e procura de condições de trabalho, a decisão e fixação de metas e autoavaliação. $\mathrm{O}$ factor 4 corresponde às "estratégias cognitivas de aquisição e selecção da informação" (ECASI, quatro 
itens), relacionadas com a atenção e participação nas aulas e procura de esclarecimentos sobre a informação junto dos docentes. Finalmente, o factor 5 corresponde às "estratégias afectivas de reforço motivacional" (EARM) e inclui dois itens que focam o interesse e a motivação na frequência às aulas. O coeficiente de consistência interna (alpha de Cronbach) é de 0,85 (Bessa \& Tavares, 2001). Na amostra utilizada neste estudo o seu valor é respectivamente de 0,83 (nível inicial) e 0,82 (nível intermédio e final).

\section{Procedimentos}

Para a realização do estudo obtiveram-se as autorizações dos directores de curso. Foram apresentados aos alunos os objectivos do estudo, sendo referido que o mesmo se inscrevia num dos projectos de investigação do Centro de Investigação em Psicologia da Universidade do Minho. Foi garantida a confidencialidade das respostas, sendo obtido o consentimento informado dos participantes. O preenchimento era voluntário e os alunos foram informados que a não participação não teria quaisquer implicações. Dos presentes nas aulas em que decorreu a aplicação dos instrumentos, todos aceitaram participar. A aplicação foi efectuada de acordo com as instruções e sempre pelo mesmo investigador. A aplicação dos questionários foi colectiva e decorreu numa única sessão por ano e curso, nos contextos das respectivas aulas práticas. A opção pela aplicação dos questionários nestas aulas prendeu-se com o facto da assiduidade dos alunos ser controlada, existindo um número máximo de faltas que o aluno pode dar. A superação desse máximo determina a sua reprovação. Este facto origina uma participação dos alunos na ordem dos $90 \%$. O número de alunos por grupo/turma variou entre 19 e 40 alunos, reflectindo o número máximo de candidatos admitidos em cada curso. O tempo médio de resposta ao questionário rondou os 20 minutos.

\section{Resultados}

A análise das diferenças nas estratégias cognitivas de transformação e manipulação da informação (ECTMI), organização e planeamento de rotinas (ECOPR), gestão e monitorização (ECGM), aquisição e selecção da informação (ECASI), reforço motivacional (EARM), em função do nível de ensino e da área académica dos alunos, foi estudada recorrendo à análise de variância multivariada. Previamente ao seu cálculo, procedeu-se à verificação da normalidade da distribuição, da homogeneidade da variância e das correlações entre as variáveis dependentes consideradas. O teste de Kolmogorov-Smirnoff para os alunos de letras e de ciências do grupo inicial, intermédio e final é estatisticamente significativo, pelo que se rejeita a hipótese da normalidade da distribuição. O teste $\mathrm{M}$ de Box mostra que as matrizes de covariância das variáveis dependentes nos grupos são iguais (Box'M=88,51; $F(2,75)=1,14 ;$ Sig.=0,175). O teste de Levene para as variáveis ECTMI $\left(F_{(5,511)}=0,49 ; p=0,782\right)$, ECOLR $\left(F_{(5,211)}=0,51 ; p=0,77\right)$, ECMGO $\left(F_{(5,211)}=1,06 ; p=0,38\right)$; $\operatorname{ECASI}\left(F_{(5,211)}=1,05 ; p=0,39\right), \operatorname{e~EARM}\left(F_{(5,211)}=1,16 ; p=0,32\right)$ indica que se pode assumir a igualdade das variâncias nos grupos considerados. O padrão de correlações encontrado indica que as cinco estratégias apresentam correlações moderadas entre si (cf. Tabela 3).

Tabela 3. Descrição das correlações entre as cinco estratégias autoreguladoras da aprendizagem.

\begin{tabular}{lllll}
\hline & ECTMI & ECOPR & ECGM & ECASI \\
\cline { 2 - 5 } ECOPR & $0,57^{*}$ & & & \\
ECGM & $0,44^{*}$ & $0,45^{*}$ & & \\
ECASI & $0,57^{*}$ & $0,40^{*}$ & $0,35^{*}$ & \\
EARM & $0,12^{*}$ & $-0,6$ & 0,03 & $0,13^{*}$ \\
\hline
\end{tabular}

Tabela 4. Estatística descritiva na amostra global e em função da área e nível.

\begin{tabular}{|c|c|c|c|c|c|c|c|c|}
\hline & & \multirow[b]{2}{*}{ Amostra Global } & \multicolumn{3}{|c|}{ Ciências } & \multicolumn{3}{|c|}{ Letras } \\
\hline & & & Nível Inicial & Nível Interm. & Nível Final & Nível Inicial & Nível Interm. & Nível Final \\
\hline & $M$ & 21,73 & 22,3 & 21,17 & 21,18 & 22,15 & 22,59 & 21,54 \\
\hline & $D P$ & 4,71 & 5,39 & 4,43 & 4,9 & 4,54 & 4,88 & 4,29 \\
\hline \multirow{3}{*}{ ЕСТМI } & Amp. & $7-34$ & $8-33$ & $8-33$ & $8-34$ & $10-32$ & $7-31$ & $8-30$ \\
\hline & Assim. & $-0,42$ & $-0,39$ & $-0,41$ & $-0,32$ & $-0,39$ & $-0,78$ & $-0,42$ \\
\hline & Curt. & 0,31 & 0,41 & 0,06 & 0,71 & 0,35 & 0,57 & 0,19 \\
\hline \multirow{6}{*}{ ECOPR } & $M$ & 20,01 & 20,43 & 19,73 & 19,03 & 20,47 & 20,55 & 20,46 \\
\hline & $\mathrm{DP}$ & 3,23 & 3,09 & 3,07 & 3,37 & 2,88 & 3,44 & 3,22 \\
\hline & Amp. & $6-25$ & $12-25$ & $10-25$ & $11-25$ & $14-25$ & $6-25$ & $9-25$ \\
\hline & Assim. & $-0,64$ & $-0,741$ & $-0,47$ & $-0,34$ & $-0,31$ & $-1,23$ & $-0,85$ \\
\hline & Curt. & 0,47 & $-0,19$ & 0,18 & $-0,47$ & $-0,49$ & 2,71 & 1,29 \\
\hline & $M$ & 25,71 & 25,52 & 25,11 & 24,28 & 26,66 & 26,68 & 26,65 \\
\hline \multirow{4}{*}{ ECMGM } & $D P$ & 3,3 & 3,46 & 2,87 & 3,7 & 3,25 & 2,84 & 2,98 \\
\hline & Amp. & $12-35$ & $15-32$ & $19-31$ & $12-32$ & $18-35$ & $17-33$ & $18-33$ \\
\hline & Assim. & $-0,46$ & $-0,5$ & 0,12 & $-0,68$ & $-0,36$ & $-0,29$ & $-0,48$ \\
\hline & Curt. & 0,63 & 0,84 & $-0,55$ & 1,09 & 0,01 & 0,23 & 0,4 \\
\hline \multirow{6}{*}{ ECASI } & $M$ & 13,38 & 13,72 & 13,4 & 13,18 & 13,13 & 13,88 & 13,03 \\
\hline & $D P$ & 2,82 & 3,04 & 2,45 & 2,95 & 2,77 & 2,86 & 3,09 \\
\hline & Amp. & $6-20$ & $6-20$ & $7-19$ & $6-20$ & $6-20$ & $6-20$ & $6-20$ \\
\hline & Assim. & $-0,08$ & $-0,29$ & $-0,06$ & 0,14 & $-0,05$ & $-0,13$ & $-0,06$ \\
\hline & Curt. & $-0,04$ & 0,57 & $-0,09$ & 0,01 & 0,24 & $-0,83$ & $-0,13$ \\
\hline & $M$ & 5,62 & 5,35 & 5,95 & 5,4 & 5,59 & 5,87 & 5,21 \\
\hline \multirow{4}{*}{ EARM } & $D P$ & 2,3 & 2,45 & 2,21 & 2,06 & 2,23 & 2,46 & 2,52 \\
\hline & Amp. & $1-10$ & $1-10$ & $2-10$ & $1-10$ & $2-10$ & $2-10$ & $2-10$ \\
\hline & Assim. & 0,03 & 0,00 & 0,00 & 0,18 & $-0,06$ & $-0,13$ & 0,28 \\
\hline & Curt. & 0,21 & $-0,89$ & $-0,81$ & $-0,47$ & $-0,62$ & $-0,83$ & 0,57 \\
\hline
\end{tabular}


Embora não se tenha verificado o pressuposto da normalidade na distribuição dos resultados, é conhecido que o teste F é robusto a violações de normalidade (Pestana \& Gageiro, 2003), pelo que é possível prosseguir com a análise do modelo. Na Tabela 4 apresentam-se as estatísticas descritivas.

$\mathrm{O}$ design factorial usado neste estudo foi do tipo $2 \times 3$ (curso x nível de frequência), pelo que é necessário proceder à análise dos efeitos principais associados ao curso e nível de frequência no ensino superior, e os efeitos de interacção "curso x nível de frequência". Na análise desta interacção, verifica-se que o nível de significância estatístico obtido $\left(V=0,023, F_{(5,511)}=1,16, p=0,32\right)$ é superior ao nível crítico habitualmente considerado $(p<0,05)$, não se verificando uma interacção significativa entre os dois factores. Na Tabela 5 descrevem-se os valores de $F$.

Tabela 5. Análise dos efeitos principais e interacções nas cinco variáveis.

\begin{tabular}{lcccccc}
\hline Efeitos & & ECTMI & ECOPR & ECMGM & ECASI & EARM \\
\hline \multirow{4}{*}{ Curso } & MQ & 53,85 & 33,35 & 27,68 & 24,13 & 3,44 \\
X & Res & 26,93 & 16,67 & 13,84 & 12,06 & 1,72 \\
Nível & $F$ & 1,22 & 1,64 & 1,37 & 1,53 & 0,33 \\
& $p$ & 0,29 & 0,19 & 0,26 & 0,22 & 0,72 \\
& $\eta^{2}$ & 0,005 & 0,006 & 0,005 & 0,006 & 0,001 \\
\cline { 2 - 7 } Nível & MQ & 54,84 & 35,88 & 29,73 & 23,66 & 36,64 \\
& Res & 27,42 & 17,94 & 14,87 & 11,83 & 18,32 \\
& $F$ & 1,24 & 1,76 & 1,47 & 1,49 & 3,47 \\
& $p$ & 0,29 & 0,17 & 0,23 & 0,23 & 0,03 \\
& $\eta^{2}$ & 0,005 & 0,007 & 0,006 & 0,006 & 0,013 \\
\cline { 2 - 7 } Curso & MQ & 34,99 & 67,73 & 332,42 & 1,29 & 0,02 \\
& Res & 34,99 & 67,73 & 332,42 & 1,29 & 0,2 \\
& $F$ & 1,58 & 6,65 & 32,92 & 0,16 & 0,00 \\
& $p$ & 0,21 & 0,01 & 0,00 & 0,69 & 0,95 \\
& $\eta^{2}$ & 0,003 & 0,01 & 0,06 & 0,00 & 0,00 \\
\hline
\end{tabular}

A diferença nos resultados em função do curso é favorável aos alunos de letras nas variáveis ECMGM e ECOPR, verificando-se que o significado estatístico das diferenças nos resultados é inferior a 0,01. Considerando a variável nível, verifica-se que diferenças estatisticamente significativas apenas se registam nas EARM. No nível inicial, a média dos resultados situa-se em 5,5 $(D P=2,30)$, no nível intermédio em $5,9(D P=2,31)$ e no nível final em 5,3. $(D P=2,25)$. Embora nestas variáveis se registem diferenças estatisticamente significativas, o valor de eta quadrado é, nas três variáveis, muito baixo. A percentagem de variância explicada varia entre $1 \mathrm{e}$ $6 \%$. Estes valores sugerem que as diferenças observadas não são substantivas. O facto do valor de significância se situar abaixo do nível crítico de 0,05 poderá dever-se à dimensão da amostra utilizada.

\section{Discussão e Conclusão}

As exigências colocadas aos alunos universitários em termos de aprendizagem requerem que os mesmos utilizem estratégias cognitivas e metacognitivas, que se apresentam como fulcrais para o sucesso académico. $\mathrm{O}$ confronto com tarefas que requerem quer a utilização eficiente de estratégias de aprendizagem quer elevados níveis de autonomia poderá conduzir, ao longo dos anos, a mudanças no padrão de es- tratégias auto-regulatórias do sujeito. Cada curso do ensino superior, por seu lado, pode privilegiar o desenvolvimento de certo tipo de estratégias em detrimento de outras. $\mathrm{O}$ estudo destas mudanças em diferentes tipos de estratégias autoreguladoras da aprendizagem constituiu o objectivo de estudo neste trabalho. Os resultados obtidos apontam no sentido da não-ocorrência de mudanças substantivas em função do ano e da área de formação, ou seja, no geral os alunos da amostra, independentemente da área de formação e do ano em que se encontram, utilizam o mesmo tipo de estratégias auto-reguladoras da aprendizagem. Outras investigações têm chegado a conclusões não coincidentes com esta. Resultados estatisticamente significativos entre alunos do $1^{\circ}$ e $4^{\circ}$ anos dos cursos de letras e ciências são referidas por Chaleta, Grácio e Rosário (2005) em algumas estratégias cognitivas ligadas à memorização e compreensão e metacognitivas (planificação do estudo, alteração da forma de estudar e verificação da aprendizagem). Suárez, Buey e Díez (2000) obtiveram diferenças significativas entre os alunos de humanidades quando comparados com os de outros cursos. Porém, nestes trabalhos não são apresentados resultados relativos à magnitude do efeito, pelo que se torna difícil apreciar que proporção de variância é atribuída ao efeito. Hofer, Yu e Pintrich (1998), a propósito de estudos que realizaram com alunos universitários, sobre a eficácia de programas de ensino de estratégias auto-regulatórias referem que as estratégias motivacionais são fundamentais uma vez que facilitam/favorecem a utilização das estratégias cognitivas e auto-regulatórias. Os mesmos autores referem ainda que as estratégias cognitivas associadas à organização do estudo (tirar notas, parafrasear, sumariar) e as estratégias metacognitivas de planificação, monitorização e regulação do estudo se revestem de uma grande importância para o bom desempenho dos alunos. Por sua vez, Garavalia e Grendler (2002) referem que os alunos utilizam com mais frequência estratégias cognitivas, nomeadamente tirar notas, em detrimento de estratégias associadas à procura de informação.

Os resultados obtidos suscitam uma discussão importante sobre a utilização de estratégias auto-reguladoras da aprendizagem, uma vez que a possibilidade de existirem diferenças por áreas de formação e por níveis de frequência não foi confirmada. Como referem Peverly e cols. (2003), os alunos universitários apresentam algumas dificuldades ao nível do conhecimento metacognitivo, nomeadamente no que se relaciona com as tarefas. $\mathrm{O}$ facto de não se observarem diferenças ao longo dos anos coloca questões importantes. Como explicar a inexistência de mudanças significativas? Qual o significado das mesmas?

Estes resultados poderão sugerir que no seu percurso académico os alunos construíram um reportório pessoal de estratégias auto-reguladoras, o que poderá originar uma maior dificuldade em adquirir novas estratégias ou alterar as que já utilizam durante o ensino superior (Hofer \& cols., 1998). Da revisão de estudos efectuada pode-se constatar que aqueles têm procurado analisar mais a relação entre estratégias auto-regulatórias e rendimento escolar e menos as mudanças ocorridas ao longo do ensino superior. O design seguido, transversal, não permite discutir questões relacionadas com a evolução na utilização de estratégias auto-reguladoras da aprendizagem ao longo dos anos. Um estudo de cariz longi- 
tudinal apresenta-se como uma alternativa a considerar em pesquisas posteriores.

Os resultados desta investigação encontram-se afectados por dois tipos de factores: os participantes e o instrumento utilizado na investigação. Apenas foram avaliados os sujeitos que estavam presentes nas aulas. $\mathrm{O}$ recurso a este procedimento, embora habitual, exclui um pequeno grupo de alunos. O significado a atribuir a esta situação é difícil de definir, mas pode colocar algumas questões em termos de representatividade, uma vez que estes alunos são percepcionados como sendo aqueles que têm uma maior probabilidade de regulação (Bessa, 2006). Esta situação poderá ser revista em futuras investigações, nomeadamente através da aplicação dos instrumentos por outras vias que não apenas a presencial (preenchimento dos questionários em papel durante a aula), bem como a sua recolha no momento em que os alunos fazem a renovação da sua matrícula. Quanto ao instrumento utilizado, é possível que o mesmo possa não abranger todos os aspectos relacionados com comportamento/estratégias auto-regulatórias. O último factor em particular pode suscitar algumas dúvidas, tendo os próprios autores (Bessa \& Tavares, 2001) apontado para a necessidade de se rever alguns dos tópicos. Uma análise mais detalhada aos itens que compõem o questionário poderá revelar-se importante em futuras investigações. Os questionários de auto-relato, como o usado, podem induzir respostas socialmente desejáveis. A possibilidade de recurso a entrevistas semi-estruturadas pode constituir uma alternativa de validação dos dados.

O CHE (Bessa \& Tavares, 2001) apenas foi utilizado, até ao momento, em dois estudos efectuados em Portugal, pelo que seria interessante replicar este estudo noutros países de língua oficial portuguesa e confrontar os resultados.

Os dados obtidos colocam questões relevantes do ponto de vista da intervenção. A verificar-se uma estabilidade nos padrões de utilização de estratégias auto-regulatórias importa determinar se tal estabilidade tem um efeito positivo ou negativo, no sentido em que os mesmos podem ser ou não adequados e de que modo se pode promover a mudança de padrões desajustados.

\section{Referências}

Almeida, L. (2001). Acesso, integração e sucesso académico: Uma análise reportada aos estudantes do $1^{\circ}$ ano. Em R. Sousa., E. Sousa., F. Lemos \& C. Januário (Orgs.), Pedagogia na Universidade: Simpósio da Universidade Técnica de Lisboa (pp. 223-240). Lisboa: Universidade Técnica de Lisboa.

Bessa, J. \& Tavares, J. (2001). Níveis de Ajustamento e autoregulação académica em Estudantes do $1^{\circ}$ ano (comum) de Ciências e Engenharias da Universidade de Aveiro. Em J. Tavares \& R. Santiago (Orgs), Ensino superior. (In)sucesso académico (pp. 107-132). Porto: Porto Editora.

Bessa, J. A. (2000). Níveis de ajustamento e auto-regulação académica em estudantes universitários: estudo com alunos do $1^{o}$ ano (comum) das licenciaturas de ciências e engenharias da Universidade de Aveiro. Dissertação de Mestrado, Universidade de Aveiro, Aveiro

Bessa, J. A. (2006). Aprendizagem auto-regulada, envolvimento e ganhos académicos em estudantes do ensino superior. Tese de Doutoramento, Universidade de Aveiro, Aveiro.
Borkowsky, J. G., Carr, M., Rellinger, E. \& Pressley, M. (1990). Self-regulated cognition: interdependence of metacognition, attributions, and self-esteem. Em B. Jones \& L. Idol (Orgs.), Dimensions of thinking and cognitive instruction (pp. 53-92). New Jersey: Lawrence Erlbaum Associates.

Brown, A. L. \& Day, J. D. (1983). Macrorules for summarizing text: The development of expertise. Journal of Verbal Learning and Verbal Behavior, 22, 1-14.

Brown, A. L., Day, J. D. \& Jones, R. S. (1983). The development of plans for summarizing texts. Child Development, 54, 968979.

Chaleta, M. E., Grácio, M. L. \& Rosário, P. (2005). Estratégias de aprendizagem em alunos universitários. Em B.D. Silva \& L. S. Almeida (Orgs.), Actas VIII Congresso Galaico Português de PsicoPedagogia (pp. 1913-1922). Braga: CIEd-UM.

Dewitte, S. (2000). Procrastinators lack a broad action perspective. European Journal of Personality, 2(14), 121-140.

Ferrari, J. (2001). Procrastination as self-regulation failure of performances: Effects of cognitive load, self-awareness, and time limits on working best under pressure. European Journal of Personality, 15, 391-406.

Garavalia, L. \& Grendler, M. (2002). An exploratory study of academic goal setting, achievement calibration and selfregulated learning. Journal of Instructional Psychology, 29(4), 221-230.

Hofer, B., Yu, S. \& Pintrich, P. (1998). Teaching college students to be self-regulated learners. Em D. Schunk \& B. Zimmerman (Orgs.), Self-regulated learning: from teaching to self-reflective practice. (pp 57-85). New York: The Guilford Press.

Howell, A. \& Watson, D. (2007). Procrastination: Associations with achievement goal orientation and learning strategies. Personality and Individual Differences, 43, 167-178.

Howell, A., Watson, D., Powell, R. \& Buro, K. (2006). Academic procrastination: The pattern and correlates of behavioural postponement. Personality and Individual Differences, 40, 1519-1530.

Pestana, M. \& Gageiro, J. (2003). Análise de dados para ciências sociais. A complementaridade do SPSS. Lisboa: Edições Sílabo.

Peverly, S. T., Brobst, K. E., Graham, M. \& Shaw, R. (2003). College adults are not good at self- regulation: A study on the relationship of self-regulation, note-taking, and test taking. Journal of Educational Psychology, 95(2), 335-346.

Pintrich, P. R. (2000). The role of goal orientation in self-regulated learning. Em M. Boekaerts, P. R. Pintrich \& M. Zeidner (Orgs.), Handbook of self-regulation (pp. 452-502). San Diego: Academic Press.

Pressley, M. \& Ghatala, E. S. (1990). Self-regulated learning: Monitoring learning from text. Educational Psychologist, 25, 19-33.

Pressley, M., Ghatala, E. S., Woloshyn, V. \& Pirie, J. (1990). Sometimes adults miss the main ideas and do not realize it: Confidence in responses to short answer and multiple-choice comprehension questions. Reading Research Quarterly, 25, 233-249.

Read, S. (1999). Commentary: characteristics of assessment in support of student access and success. Em S. Messick (Org.), Assessment in higher education: issues of access, quality, student development, and public policy (pp. 63-68). London: Lawrence Erlbaum Associates Publishers. 
Santos, L. (2001). Adaptação académica e rendimento escolar: Estudo com alunos universitários do $1^{\circ}$ ano. Dissertação de Mestrado, Universidade do Minho, Braga.

Suárez, F., Buey, F. \& Diez, J. (2000). Estilos y estrategias de aprendizaje en estudiantes universitários. Psicothema, 12, 4, 615-622.

Wolters, C. (1998). Self-regulated learning and college students' regulation of motivation. Journal of Educational Psychology, 90(2), 224-235.

Zimmerman, B. J. (1994). Dimensions of academic self-regulation: A conceptual framework for education. Em B. J. Zimmerman \& D. H. Schunk (Orgs), Self-regulation of learning and performance: issues and educational applications (pp. 3-21). New Jersey: Lawrence Erlbaum Associates.

Zimmerman, B. J. (1998). Developing self-fulfilling cycles of academic regulation: An analysis of exemplary instructional models. Em D. H. Schunk \& B. J. Zimmerman (Orgs.), Selfregulated learning: from teaching to self-reflective practice (pp. 1-19). New York: The Guilford Press.
Zimmerman, B. J. (2001). Theories of self-regulated learning and academic achievement: an overview and analysis. Em B. J. Zimmerman \& D. H. Schunk (Orgs.), Self-regulated learning and academic achievement: theoretical perspectives (pp. 1-38). New Jersey: Lawrence Erlbaum Associates.

Zimmerman, B. J. \& Martinez-Pons, M. (1992). perceptions of efficacy and strategy use in the self-regulation of learning. Em D. H. Schunk \& J. Meece (Orgs.), Student perceptions in the classroom (pp. 185-207). New Jersey: Lawrence Erlbaum Associates.
Recebido em 07.02.2006

Primeira decisão editorial em 30.05.2006

Versão final em 15.06.2007

Aceito em 20.08.2007 